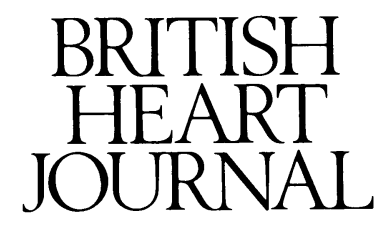

\title{
Editorial
}

\section{Biomagnetometry: imaging the heart's magnetic field}

Make a fist with your right hand, let go slightly, and stick your thumb in the air. You are now illustrating the "right hand rule", which states that if the thumb points in the direction of electric current flow the curl of the fingers indicates the direction of the magnetic field. Hans Oersted, in 1819, was the first to show that whenever an electric current flows it produces a magnetic field at right angles to the direction of the current. The problem in trying to measure the magnetic fields produced by electrically active parts of the body such as the brain and the heart is that these fields are exceedingly weak. The field strength at the surface of the body is between $10^{-11} \mathrm{~T}$ and $10^{-14} \mathrm{~T}$ compared with the earth's magnetic field of about $10^{-4} \mathrm{~T}$. Research and development by basic scientists undaunted by these problems have resulted in commercial biomagnetometers that are completely non-invasive and have excellent temporal resolution and reasonably good spatial resolution.

In 1963 Baule and McFee published a recording of a human magnetocardiogram that they obtained using two large induction coils, each consisting of 2 million windings, positioned over the chest. ${ }^{1}$ The record looked like a noisy derivative of the electrocardiogram. The magnetocardiogram may be subjected to filtering and signal averaging but the information obtained is not as good as that in a signal averaged electrocardiogram because of the inherently smaller signal. Measurements of magnetic fields can, however, provide better spatial resolution than electric currents because they are not distorted by flow through the tissues. There is significant and varying resistance in the different tissues of the body to electric current flow but the body is transparent to low frequency magnetic fields.

The recording head of a biomagnetometer lies in a plane that is normal to the surface of the body and a signal can be measured providing that the dipole source of the magnetic field is eccentric in the body and is orientated predominantly tangentially to the body surface. To make a map of the heart's magnetic field more recording sites are necessary, which means that the recording heads need to be small. The problem of how to get measurable signals from small detection coils has been overcome by the development of a low noise amplifier known as a superconducting quantum interference device (or SQUID for short). Within the recording head the detection coils and the SQUID are cooled to $4^{\circ} \mathrm{C}$ above absolute zero $\left(-269^{\circ} \mathrm{C}\right)$ by liquid helium in an evacuated container. The amplified signals are digitised and processed by computer. Isomagnetic contour maps of the changing magnetic fields under the sensor heads can be displayed on a video screen and printed out. The first human magnetocardiogram obtained with a SQUID was recorded in $1969 .^{2}$ The SQUID became available commercially in 1970 and the next year the first magnetocardiogram map was recorded. ${ }^{3}$

From these maps of magnetic field strength it is possible to calculate the position of an electric current source that would produce the magnetic contour patterns observed and, depending on the model used, this point may be called an equivalent current dipole. The position of this electrical dipole may be superimposed over a magnetic resonance image (separately obtained) to show the position of the apparent current source that gives rise to the magnetic field. At this point it is worth stating the distinction between magnetic resonance imaging and biomagnetometry. Magnetic resonance imaging measures proton magnetic resonance in an applied magnetic field and gives information that is anatomically useful. Biomagnetometry gives physiological information about the magnetic fields generated by the individual. Indeed a substantial part of the very high cost of a biomagnetometry system goes to housing the non-magnetic equipment in a shielded room to minimise interference from extraneous magnetic and electrical fields and from mechanical vibration.

The simple shape of the human head makes localisation of current sources in the brain more straightforward than in the heart and the technique has great potential in neurology for the localisation of epileptic foci and other electrical events that leave no trace on computed tomograms or magnetic resonance images. Computer reconstruction of the source(s) of the magnetic signals from the heart is complicated by the difficulty of modelling the heart's moving geometry and also because the magnetic signal at the surface of the body is affected by current flow between individual cardiac myocytes. ${ }^{4}$ Nevertheless, with an isomagnetic map it is possible to derive an equivalent current dipole corresponding to, for example, the onset of ventricular depolarisation. The spatial accuracy of the technique is illustrated by finding that in patients with a ventricular pacemaker the equivalent current dipole is located within $1.1 \mathrm{~cm}$ of the position of the electrode tip. ${ }^{5}$

Similarly, the sources of extrasystoles ${ }^{67}$ and the positions of accessory pathways ${ }^{8}$ can be plotted and good correlation has been found between the sites of accessory pathways recorded directly and those derived from biomagnetometry. The spatial resolution of the technique theoretically approaches that of magnetic resonance imaging, which is approximately $2 \mathrm{~mm}$. Some data are available from earlier biomagnetometers consisting of 1-7 recording channels and these suggest that the accuracy of three dimensional localisation of accessory pathways is in the range $3 \mathrm{~mm}-5 \mathrm{~cm}$ with these machines. The latest generation of machines available in 1990 use 45 recording units, 37 of which are for data collection and eight for noise 
detection, and it is expected that the accuracy of localisation of derived current sources will improve correspondingly. Another benefit of having more detectors is that the time necessary for the examination has been reduced. It can now be completed in 10-20 minutes rather than taking up to several hours.

The isomagnetic contour maps may eventually have a wider usefulness than the derived information on the positions of current sources. The spatial distribution of the magnetic field contours does not normally vary very much when the net electrical activity of the heart is relatively quiescent, for example during the ST segments of the electrocardiogram. In patients after acute myocardial infarction the magnetic contour maps taken at intervals of 25 ms may change markedly during the ST segment, ${ }^{7}$ and the maps may potentially provide much more information than signal averaged electrocardiograms recorded during this period. An index has been derived of the "relative smoothness" of the magnetic fields during the course of the ST segment ${ }^{9}$ and this index seems to be significantly reduced in patients with ventricular arrhythmias caused by factors such as cardiomyopathy. Though only small numbers of patients have been studied so far, it has been suggested from data such as these that biomagnetometry may be useful in identifying patients at high risk of developing serious ventricular arrhythmias. It would be fascinating to know the effects of antiarrhythmic drugs (successful or otherwise) on the isomagnetic maps in patients with ventricular arrhythmias and on patterns of current flow through accessory pathways. And research interest need not be restricted to the study of arrhythmias; it is likely that the magnetic patterns are significantly altered in, for example, haemochromatosis affecting the heart.

With the new generation of machines, biomagnetometry has become an important clinical research tool and machines are available from Biomagnetic Technologies, San Diego, costing about $\$ 2$ million. Siemens have also developed a 37 channel system and other manufacturers have active research programmes. The encouraging results from machines with 2,7 , and 14 channels have prompted this new generation of 37 channel units and we wait to see what sort of resolution they will have, but it may be necessary to have more than 100 SQUIDs before biomagnetometry can reliably challenge invasive electrical mapping for localisation of the site of origin and the conduction pathways of arrhythmias. These new machines already have the potential to identify patients who are at high risk from arrhythmias after myocardial infarction and to monitor the effects of antiarrhythmic medication better than we can at the moment.

The next stage for investigators is to define the role (if any) of biomagnetometry in clinical cardiac practice. For those who have access to a biomagnetometer it will be important to assess the spatial accuracy of the new generation of machines; to correlate findings on accessory pathways and arrhythmogenic foci with data from conventional electrophysiological studies; to study a large cohort of patients after myocardial infarction by contour mapping, correlating magnetic activity during the ST segment with sudden death, arrhythmias, and other complications; and to investigate the patterns of electrical activity in patients with cardiomyopathies, etc. Only by careful clinical trials with adequate numbers of patients can the potential value of this promising technique be properly assessed and criteria drawn up on which to base its routine clinical use.

Department of Cardiovascular Medicine, G HART University of Oxford,

John Radcliffe Hospital, Oxford OX3 9DU

1 Baule GM, McFee R. Detection of the magnetic field of the heart. Am Heart $J$ 1963;66:95-6.

2 Cohen D, Edelsack A, Zimmerman JE. Magnetocardiograms taken inside a shielded room with a superconducting point-contact magnetometer. App Phys Lett 1970;16:278-80.

3 Cohen D, McCaoughan D. Magnetocardiograms and their variation over the chest in normal subjects. Am J Cardiol 1972;29:678-85.

4. Williamson SJ, Romani GL, Kaufman L, Modena I, eds. Biomagnetism : an interdisciplinary approach. New York: Plenum, 1983.

5 Fenici RR, Melillo G, Cappelli A, De Luca C, Masselli M. Magnetocardiographic localization of a pacing catheter. In: Biomagnetism '89. Tokyo: Tokyo Denki University Press, 1990 (in press).

6 Fenici RR, Masselli M, Lopez L, Melillo G. Magnetocardiographic localization of arrhythmogenic tissue. In: Atsumi K, Kotani M, Ueno S, Katila T, Williamson SJ, eds. Biomagnetism '87. Tokyo: Tokyo Denki Katila T, Williamson SJ, eds.
University Press, 1988:282-5.

7 Schmitz L, Oeff M, Erne SN. Localization of arrhythmogenic areas in the human heart. In: Atsumi K, Kotani M, Ueno S, Katila T, Williamson SJ, eds. Biomagnetism '87. Tokyo: Tokyo Denki University Press, 1988: 286-9.

8 Nomura M, Nakaya Y, Watanabe K, et al. Detection of accessory pathway in patients with WPW syndrome by means of the isomagnetic map and MRI. In: Biomagnetism '89. Tokyo: Tokyo Denki University Press, 1990 (in press).

9 Schmitz L, Brockmeier K, Trahms L, Erne SN. Magnetocardiography in patients with cardiomyopathy and operated congenital heart disease. In: Biomagnetism '89. Tokyo: Tokyo Denki University Press, 1990 (in press). 